\title{
ESR AND ELECTRON SPIN ECHO STUDIES OF SPIN-LATTICE RELAXATION OF HYDRAZINIUM RADICAL IN $\mathrm{Li}\left(\mathrm{N}_{2} \mathrm{H}_{5}\right) \mathrm{SO}_{4}$ SINGLE CRYSTAL
}

\author{
P. Morawski, S.K. Hoffmann, W. Hilczer and J. Goslar \\ Institute of Molecular Physics, Polish Academy of Sciences \\ Smoluchowskiego 17, 60-179 Poznań, Poland \\ (Received January 13, 1997; in final form April 14, 1997)
}

\begin{abstract}
A hydrazinium $\dot{\mathrm{N}} \mathrm{H}-\mathrm{NH}_{3}^{+}$radical was identified by ESR in $\boldsymbol{\gamma}$-irradiated $\mathrm{Li}\left(\mathrm{N}_{2} \mathrm{H}_{5}\right) \mathrm{SO}_{4}$ single crystal. Unpaired electron is strongly localized on the nitrogen atom as compared to hydrazinium radicals in other crystals. Angular variations of the multicomponent ESR spectra and computer calculations gave the isotropic hyperfine coupling parameters (in $\mathrm{mT}$ ): $a_{\mathrm{N} 1}=1.93$, $a_{\mathrm{N} 2}=0.80, a_{\mathrm{H} 1}=2.50, a_{\mathrm{H} 1^{\prime}}=0.48\left(\mathrm{NH}_{3}\right)$, and $a_{\mathrm{H} 2}=1.40$ (adjacent molecule). The radiation defects containing unpaired electrons do not influence much the protonic conductivity along the hydrazine molecule chains in the crystal. It confirms a tunelling mechanism of the conductivity activated by $\mathrm{NH}_{2}$-group rotation. Spin-lattice relaxation time $T_{1}$ varies strongly with temperature in the range from $100 \mathrm{~ms}(10 \mathrm{~K})$ to $90 \mu \mathrm{s}(170 \mathrm{~K})$ and is governed by spectral diffusion $\left(1 / T_{1} \propto T^{2}\right)$ and a Murphy-type mechanism $\left(1 / T_{1} \propto \operatorname{cosech}(\Delta / T)\right)$ with $\Delta=150 \mathrm{~cm}^{-1}$. A distribution of the spin-relaxation times was observed with temperature independent dispersion parameter $\beta=0.76$.
\end{abstract}

PACS numbers: $61.80 . \mathrm{Ed}, 76.30 . \mathrm{Rn}$

\section{Introduction}

Lithium hydrazinium sulphate $\mathrm{Li}\left(\mathrm{N}_{2} \mathrm{H}_{5}\right) \mathrm{SO}_{4}-$ LIIS is a fast proton conducting solid with potential applications in high energy density batteries, sensors, fuel cells and electrochemical systems [1] with implications to energy transduction in cellular biological processes [2]. The transport and dielectric properties of the crystal are highly anisotropic with proton conductivity and dielectric permittivity being of about three orders of magnitude larger along the crystal $c$-axis as compared to the $b a$-plane $[3,4]$. These one-dimensional crystal properties are related to its crystal structure. LHS is an orthorhombic crystal $\left(P n a 2_{1}\right)$ with four molecules in the unit cell $[5,6]$. The crystal structure consists of $\mathrm{LiO}_{4}$ and $\mathrm{SO}_{4}$ tetrahedrons forming a three-dimensional framework containing channels running along the $c$-axis direction. The hydrazinium ions $\mathrm{NH}_{2}-\mathrm{NH}_{3}^{+}$are located in the channels with $\mathrm{N}-\mathrm{N}$ bonds perpendicular to the channel axis. The ions are linked into 


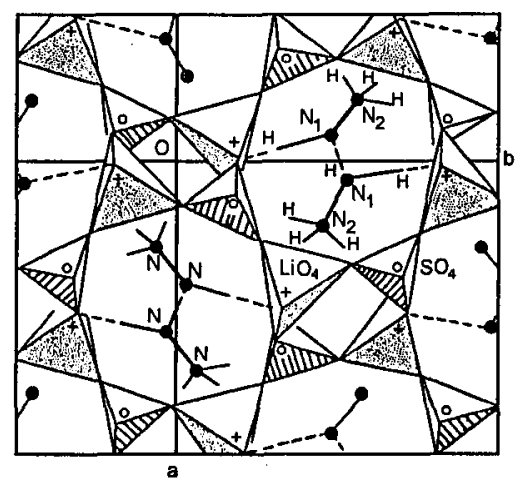

Fig. 1. Lithium hydrazinium sulphate structure projected along the c-axis. Oxygen atoms are located at the apices of the $\mathrm{LiO}_{4}$ and $\mathrm{SO}_{4}$ tetrahedra, and hydrazinium ions $\mathrm{NII}_{2}-\mathrm{NH}_{3}^{+}$lie in the channels and are connected into chains by hydrogen bonds.

chains by hydrogen bonds between $\mathrm{NI}_{2}$-groups whereas the rotating $\mathrm{NH}_{3}$-groups are hydrogen-bonded to the sulphate framework (Fig. 1).

The protonic conductivity is very fast along the hydrazinium chains with the conductivity value of $\sigma \approx 10^{-8} \Omega^{-1} \mathrm{~cm}^{-1}$ and activation energy of $E_{\mathrm{a}}<1 \mathrm{eV}$ along $c$-axis at room temperature [4, 7]. The mechanisms of the proton conductivity in LIIS are still controversial with a few proposed models. For d.c. and low frequency a.c. conductivity the charge flow is governed mainly by defects in crystal lattice $[3,8]$, whereas for higher frequencies another two mechanisms can dominate. One is a tunnelling of protons along the chains accompanied by rotation of the $\mathrm{NI}_{2}$ groups [7,9] and second is named a "vehicle" mechanism carrying the proton through the crystal lattice $[7,10]$. These two mechanisms are related to the polarizability of the molecules involved in the hydrogen bond network, rather than to a mass transport of the protons.

The macroscopic LIIS crystal properties are strongly related to the dynamical behaviour of the hydrazinium ions which was studied by vibrational Raman spectroscopy [11] and proton NMR $[12,13]$. It was found, moreover, that the crystal properties are strongly affected by ionized damage with significant reduction of the conductivity and dielectric anisotropy [14].

To deduce the structure of the free radical formed by $\gamma$-irradiation and its dynamical behaviour we have performed detailed ESR studies of single crystals and pulsed ESR studies of spin-lattice relaxation in the wide temperature range.

\section{Experimental}

LHS single crystals were grown by a slow evaporation of a saturated aqueous solution of triple recrystallized $\mathrm{Li}\left(\mathrm{N}_{2} \mathrm{H}_{5}\right) \mathrm{SO}_{4}$ at room temperature. The crystals are elongated along crystal $c$-axis with well developed $(010),(110)$ and $(011)$ faces. The free radicals were formed by $\gamma^{-60}$ Co irradiation with doses in the range $1.4-12.8 \mathrm{kGy}$ producing about $10^{16}-10^{18}$ spins/gram. The radicals are very stable and no change in the number of spins was observed over the 8 months. 
ESR spectra of powder and single crystal samples were recorded with a RADIOPAN SE/X-2547 spectrometer operating at $9.4 \mathrm{GHz}$ with a $\mathrm{TE}_{102}$ rectangular cavity and $100 \mathrm{kIIz}$ magnetic modulation, equipped with a flow helium cryostat OXFORD ESR 900. Angular variations of the single crystal spectra were recorded in the three orthogonal crystal planes by rotation of the crystal around $a, b$, and $c$ axes in the microwave cavity. ESR spectra were analyzed using SNSS and DELB computer FORTRAN programs.

Pulsed ESR experiments were performed on a BRUKER ESP380E FT/CW spectrometer equipped with a helium flow cryostat OXFORD CF935 in the temperature range $4.2-170 \mathrm{~K}$. Temperature dependence of the spin-lattice relaxation was measured for a selected crystal orientation with external magnetic field $B_{0}$ parallel to the crystal $a$-axis with the $B_{0}$ fixed at the central line position (Fig. 2). Saturation recovery methods were used and a recovery of the magnetization was detected by electron spin echo technique. The spin system was saturated by a sequence of the five 120 ns pulses with time distance $200 \mathrm{~ns}$. This picket-fence pulse sequence minimizes an influence of the spectral diffusion on $T_{1}$. However, it was impossible to saturate the line completely and the saturation of about $70 \%$ was achieved. The IIahn echo was excited by two 120 ns pulses with 200 ns interpulse distance. The spectral band width of the $120 \mathrm{~ns}$ pulse is of about $0.3 \mathrm{mT}$, thus the central line was excited only. We found that $T_{1}$ has the same value for all hyperfine lines in the spectrum.

\section{Results and discussion}

\section{1. $C W-E S R$}

ESR spectrum of a radical formed by $\gamma$-irradiation of LIIS crystal is rather complicated and contains many relatively well resolved lines at most crystal orientations. Two symmetry-related sets of spectra were observed in the three crystal planes according to the orthorhombic crystal symmetry. The spectra coincide along the crystal axes and are shown in Fig. 2. These multicomponent spectra ire symmetrical and contain odd number of resonance lines with 15 -line pattern with $0.66 \mathrm{mT}$ splitting along the $a$-axis, 47 -line pattern with splitting of $0.32 \mathrm{mT}$ along the $b$-axis, and 31-line pattern with splitting of $0.4 \mathrm{mT}$ along $c$-axis. The line splitting and line width are angular dependent. Because the line width is not much smaller than the splitting thus in the most crystal orientations the badly resolved spectra were observed. A detailed analysis of the spectra was carried out by a computer fitting of the spectra observed along the $a, b$ and $c$ axes and for these

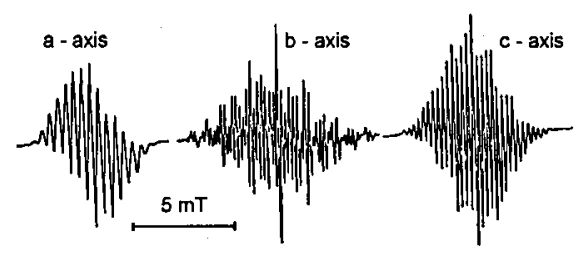

Fig. 2. ESR spectra of $\dot{\mathrm{N}} \mathrm{H}-\mathrm{NH}_{3}^{+}$radical observed along the crystallographical axes $a, b, c$ at $293 \mathrm{~K}$. 
crystal orientations where well resolved spectra were recorded. ESR spectra were simulated for different possible radical structures predicted by structural data, ESR data for other hydrazine crystals and theoretical calculations $[15,16]$ with a supposition of fast reorientations of the $\mathrm{NII}_{2}$ and/or $\mathrm{NH}_{3}$ groups. Lorentzian line shape and the same line width for all lines of a spectrum were also assumed.

Since hyperfine splittings are due to the couplings of the unpaired electron to adjacent ${ }^{1} \mathrm{H}$ and ${ }^{14} \mathrm{~N}$ nuclei the spectra have been simulated by taking into account that:

(i) the total splitting of a spectrum (distance between the utmost lines) is equal to $\sum_{i} 2 A_{\mathrm{N}_{\mathrm{i}}}+\sum_{j} A_{\mathrm{H}_{j}}-1$ in units of the smallest splitting in the spectrum (for example $0.88 \mathrm{mT}$ for $a$-axis) where $A_{\mathrm{N}}$ and $A_{\mathrm{H}}$ are hyperfine splittings for nitrogen and hydrogen, respectively;

(ii) the total spectral intensity (the sum of the amplitudes) is equal to $3^{N} \cdot 2^{H}$ in units of the lowest-field line amplitude, and $N$ and $H$ are number of interacting nitrogens and protons, respectively.

We found $N=2$ and $H=5$, with unpaired electron localized mainly on the $2 p_{x}$ orbital of the $\mathrm{N}_{1}$-nitrogen of the NII-group (Fig. 3) and local radical symmetry is lower than axial symmetry. The principal values of the $g$-tensor and hyperfine $A$-tensor components are given in Table I. The $g$-tensor axes and isotropic hyperfine parameters are shown in Fig. 3. The unpaired electron is strongly localized on the nitrogen $\mathrm{N}_{1}$ as is indicated by the largest isotropic hyperfine splitting $a_{\mathrm{N} 1}$ equal to $1.93 \mathrm{mT}$ as compared to other hydrazine-type radicals summarized in Table II, thus the ESR spectrum is dominated by isotropic contact interaction and anisotropic dipolar coupling within the NH-group.

The three protons of $\mathrm{NH}_{3}^{+}$-group give an identical hyperfine splitting confirming a fast reorientation of the group at room temperature observed in NMR experiments $[12,13]$. When temperature is lowered a continuous line broadening is observed and additional line splitting gradually appears below of about $120 \mathrm{~K}$ producing badly resolved spectra. It can be seen on the powder ESR spectra recorded at $293 \mathrm{~K}$ and $77 \mathrm{~K}$ (Fig. 4). This is due to a hindering of the $\mathrm{NH}_{3}$-group rotation at low temperatures, where the reorientation rate is too small to average the splitting from the protons, thus every proton of the $\mathrm{NH}_{3}^{+}$gives an individual hyperfine splitting.

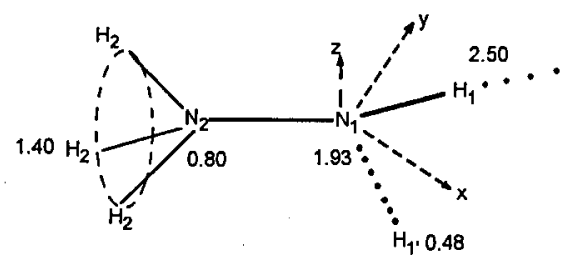

Fig. 3. Molecular structure of the hydrazinium ion radical $\mathrm{N} H-\mathrm{NH}{ }_{3}^{+}$in $\mathrm{Li}_{2}\left(\mathrm{~N}_{2} \mathrm{H}_{5}\right) \mathrm{SO}_{4}$. The isotropic hyperfine coupling constants are given in $\mathrm{mT}$. The directions $x, y, z$ of the principal $g$-tensor axes are marked: $z$-axis is perpendicular to the $\mathrm{N}_{2}-\mathrm{N}_{1}-\mathrm{H}_{1}$ plane and $x$-axis bisects the $\mathrm{H}_{1}-\mathrm{N}_{1}-\mathrm{H}_{1}$, angle. 
TABLE I

Principal values of $g$-tensor and hfs tensor of $\mathrm{N} H-\mathrm{NH}_{3}$ radical at $293 \mathrm{~K}$.

\begin{tabular}{l}
\hline \hline \multicolumn{6}{c}{$g_{z}=2.0057(5)$} & \multicolumn{1}{c}{$g_{y}=2.0039(5)$} \\
\\
\multicolumn{5}{c}{ Hyperfine coupling $[\mathrm{mT}]( \pm 0.1)$} \\
\hline
\end{tabular}

TABLE II

Isotropic spin-Hamiltonian parameters of hydrazinium and hydrazyl radicals ( $a$-values in $\mathrm{mT})^{*}$.

\begin{tabular}{|c|c|c|c|c|c|c|c|c|}
\hline Radical & $a_{\mathrm{N} 1}$ & $a_{\mathrm{N} 2}$ & $a_{\mathrm{H} 1}$ & $a_{\mathrm{H} 1^{\prime}}$ & $a_{\mathrm{H} 2}$ & $g$ & Remarks & Ref. \\
\hline $\mathrm{NH}_{3}-\dot{\mathrm{N}} \mathrm{H}$ & 1.93 & 0.80 & 2.50 & 0.48 & 1.40 & 2.0037 & $\begin{array}{l}\text { in } \mathrm{Li}\left(\mathrm{N}_{2} \mathrm{H}_{5}\right) \mathrm{SO}_{4} \\
\text { crystal }\end{array}$ & $\begin{array}{l}\text { this } \\
\text { paper }\end{array}$ \\
\hline $\mathrm{NH}_{2}-\dot{\mathrm{N}} \mathrm{II}$ & 1.17 & 0.88 & 1.88 & 2.3 & 2.3 & - & $\begin{array}{l}\text { adsorbed on } \\
\text { zeolite at }-40^{\circ} \mathrm{C}\end{array}$ & {$[17,18]$} \\
\hline $\mathrm{N}_{2} \mathrm{H}_{4}^{+}$ & 1.15 & 1.15 & 1.10 & 1.10 & - & 2.00342 & $\begin{array}{l}\text { in aqueous sulfonic } \\
\text { acid solution }\end{array}$ & [19] \\
\hline $\mathrm{N}_{2} \mathrm{H}_{4}^{+}$ & 1.44 & 1.44 & 0.89 & 0.89 & - & 2.006 & in $\mathrm{N}_{2} \mathrm{II}_{5} \mathrm{SO}_{4}$ crystal & {$[20]$} \\
\hline $\mathrm{N}_{2} \mathrm{H}_{4}^{+}$ & 1.15 & 1.15 & 1.1 & 1.1 & - & 2.0034 & in $\mathrm{N}_{2} \mathrm{H}_{6} \mathrm{SO}_{4}$ crystal & {$[21]$} \\
\hline $\mathrm{N}_{2} \mathrm{H}_{4}^{+}$ & - & - & 1.17 & 1.12 & & - & $\begin{array}{l}\mathrm{H}^{1} \text {-ENDOR of } \\
\mathrm{N}_{2} \mathrm{H}_{5} \mathrm{HC}_{2} \mathrm{O}_{4} \text { crystal }\end{array}$ & [22] \\
\hline $\begin{array}{l}\mathrm{DPPH} \\
\text { radical }\end{array}$ & 0.90 & 0.90 & - & - & - & 2.0036 & $\begin{array}{l}\text { in oxygen free } \\
\text { benzene solution at } \\
25^{\circ} \mathrm{C}\end{array}$ & {$[23]$} \\
\hline
\end{tabular}

\footnotetext{
${ }^{*} a[\mathrm{MHz}]=13.99612 g A[\mathrm{mT}]$.
} 


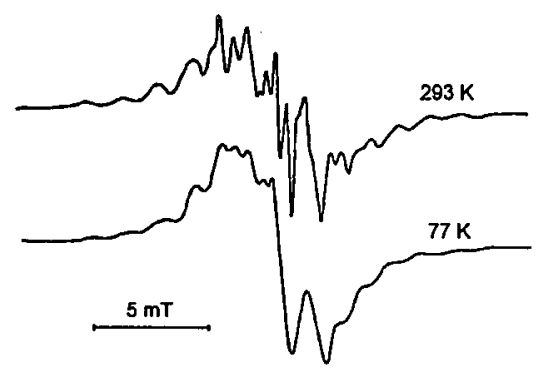

Fig. 4. ESR spectra of $\dot{\mathrm{NH}}-\mathrm{NH}_{3}$ radical in powdered $\mathrm{Li}\left(\mathrm{N}_{2} \mathrm{H}_{5}\right) \mathrm{SO}_{4}$ crystal at room and liquid nitrogen temperature.

The molecular structure of the $\dot{\mathrm{N}} \mathrm{H}-\mathrm{NH}_{3}$ radical indicates that the $\gamma$-irradiation breaks the hydrogen bond between hydrazinium ions forming the conducting chains in the crystal. Thus, one can expect a significant lowering of the proton conductivity along the chain axis ( $c$-axis) after irradiation. Unexpectedly, the effect of the radiation damage was smaller along $c$-axis than along the $a$ and $b$-axes [14]. This indicates that despite the breaking of the conducting chain the conductivity mechanism is able to maintain proton current along chain. We connect this with a dynamical behaviour of the $p_{x}$-orbital where unpaired electron is located. The $p_{x}$-orbital can be directed "downwards" or "upwards" in the hydrazinium chain. There exists a fast exchange (rotation) between these two positions leading to an avcraging of the $g$-tensor $x$-axis direction. As an effect the $x$-axis lies between two possible $\mathrm{N}-\mathrm{II}$ directions instead of along the one of these (Fig. 3). This behaviour indicates that the protonic conductivity is due to a proton tunnelling along the chains with simultaneous $\mathrm{NH}_{2}$-group reorientation.

\subsection{Spin-laltice relaxalion}

Spin-lattice relaxation time $T_{1}$ of the hydrazinium radical in $\mathrm{Li}\left(\mathrm{N}_{2} \mathrm{H}_{5}\right) \mathrm{SO}_{4}$ varies from $100 \mathrm{~ms}$ at low temperatures to about $90 \mu \mathrm{s}$ at $170 \mathrm{~K}$. Such broad range of $T_{1}$ cannot be covered by one method of measurement by saturation recovery technique. Short $T_{1}$ times were measured by standard saturation recovery technique with the IIahn echo reading scquence measuring magnetization value after saturation pulses. This technique allows to observe a saturation recovery curve for times up to $256 \mu \mathrm{s}$. This limit is set by $125 \mathrm{MIIz}$ clock of the BRUKER Pulse Programmer and $32 \mathrm{kbit}$ RAM of the Pulse Former Unit as $332 \mathrm{kbit} \times 8 \mathrm{~ns}=256 \mu \mathrm{s}$. The longer $T_{1}$ times were measured using a BRUKER software routine based on a continuous IIahn echo amplitude monitoring with varying the repetition time $t_{\mathrm{r}}$ of this sequence. Increasing the time interval between consecuting IIahn echo sequences an increase in the echo amplitude $V$ was observed. The function $V\left(t_{\mathrm{r}}\right)$ is a standard saturation recovery curve, and for $T=15 \mathrm{~K}$ is presented in Fig. 5 .

The recovery curves were not single exponential and described by stretched exponential function

$$
V(l)=V(0)+[V(\infty)-V(0)]\left[1-\exp \left(-t / T_{1}\right)^{\beta}\right]
$$




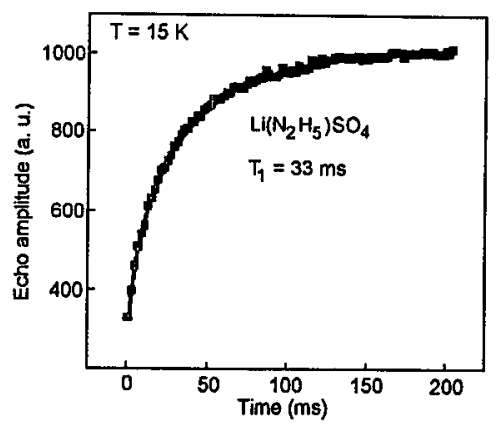

Fig. 5. The saturation recovery curve of the hydrazinium radical at $15 \mathrm{~K}$. The solid line is the fit to Eq. (1) (see text) with spin-lattice relaxation time $T_{1}=33 \mathrm{~ms}$.

where $V(0)$ is proportional to the nonsaturated part of magnetization (for $t=0$ ) and $V(\infty)$ is proportional to the equilibrium value $M_{0}$ of the magnetization for $t=\infty$. The best fit to the experimental points is presented as a solid line in Fig. 5 with $V(0)=271$ a.u., $V(\infty)=722$ a.u., $\beta=0.76$ and $T_{1}=33 \mathrm{~ms}$. Stretched exponential recovery is characteristic of a distribution of the relaxation times with mean spin-relaxation time $T_{1}$ and dispersion parameter $\beta$ [24]. We found that the dispersion parameter $\beta=0.76$ is temperature independent in our crystal. It means that the distribution of the spin-lattice relaxation times is not affected by temperature in the crystal although an increase in $\beta$-value was observed for a-Si:II [25]. We observed the temperature independent distribution of spin-lattice relaxation times with $\beta=0.70$ for glycine radical produced by $\gamma$-irradiation in diglycine nitrate crystal [26]. It seems that distribution of relaxation times is characteristic of free radicals produced by radiation damage of crystals, where the neighbourhood of radicals varies from site to site and their space distribution is nonuniform.

Temperature variations of the spin-lattice relaxation rate is shown in Fig. 6 . The best fit to these data presented by the solid line in the figure was obtained using the equation

$$
\frac{1}{T_{1}}=a T^{2}+b \operatorname{cosech}\left(\frac{\Delta}{T}\right)
$$

with parameters $a=0.128 \mathrm{~K}^{-2} \mathrm{~s}^{-1}, b=0.06 \mathrm{~s}^{-1}$ and $\Delta=216 \mathrm{~K}=150 \mathrm{~cm}^{-1}$.

The quadratic term in Eq. (2) can be due to several mechanisms and can be called a spectral diffusion term. This term contains contribution from spectral diffusion mechanism, the contributions from the two-phonon Raman processes above the Debye temperature, from a distribution of low-lying librational levels of a radical molecule, and from a distribution of the pairwise interaction between nonuniformly distributed radicals $[27,28]$.

The term of the form $\operatorname{cosech}(\Delta / T)$ in Eq. (2) frequently appearing in electron spin-lattice relaxation in organic materials can be attributed to the tunnelling rotation of the $\mathrm{NH}_{3}$-groups in adjacent molecules with tunnelling splitting $\Delta / 2=75 \mathrm{~cm}^{-1}[29]$ or to the Murphy mechanism [30]. Murphy has proposed an Orbach-Aminov type process that the absorption of a phonon causes simultaneous transition of a local vibrations of energy $\Delta$ and electron spin. This mechanism 


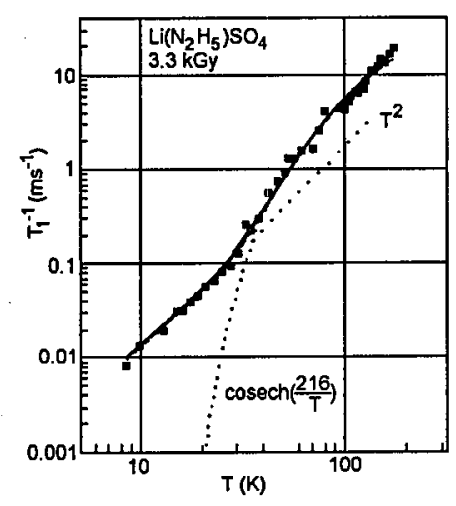

Fig. 6. Temperature dependence of the spin-lattice relaxation rate $1 / T_{1}$. The solid line is the plot fitted to the equation: $1 / T_{1}=a T^{2}+b \operatorname{cosech}(\Delta / T)$ with $\Delta=216 \mathrm{~K}=$ $150 \mathrm{~cm}^{-1}$ and $a, b$ parameters are given in the text.

was identified for $\mathrm{SeO}_{2}^{-}$radical in $\mathrm{KII}_{3}\left(\mathrm{SeO}_{3}\right)_{2}$ crystal with $\Delta_{1}=52 \mathrm{~cm}^{-1}$ and $\Delta_{2}=123 \mathrm{~cm}^{-1}[31]$.

Equation (2) does not contain terms describing a direct coupling to the phonon of the crystal lattice i.e. direct process term and Raman term. Trials to fit the data with the Raman-type term gave much worse results than fitting to Eq. (2). Thus, the electron-phonon couplings are overdominated in the whole temperature range by the local crystal dynamics and interactions within the electron-spin system.

\section{References}

[1] S. Chandra, Superionic Solids - Principles and Applications, North-Holland, Amsterdam 1981.

[2] E. Racker, A New Look at Mechanisms in Bioenergetics, Academic Press, New York 1976.

[3] V.II. Schmidt, J.E. Drumheller, F.L. Howell, Phys. Rev. 134, 4582 (1971).

[4] F.E. Salman, B. Hilczer, C. Pawlaczyk, Jpn. J. Appl. Phys. 24, 668 (1985).

[5] I.D. Brown, Acta Crystallogr. 17, 654 (1964).

[6] M.R. Anderson, I.D. Brown, Acta Cryslallogr. B 30, 831 (1974).

[7] K.D. Kreuer, W. Weppner, A. Rabenau, Solid State Ionics 3/4, 353 (1981).

[8] V.H. Schmidt, R.S. Parker, J. Phys. (France) Suppl. 33, C2-10 (1972).

[9] A. Kawada, A.R. McGhie, M.M. Labes, J. Chem. Phys. 52, 3121 (1970).

[10] K.D. Kreuer, A. Rabenau, W. Weppner, Angew. Chem. Int. Ed. Engl. 21, 208 (1982).

[11] S.H. Brown, R. Frech, Spectrochim. Acta A 44, 1 (1988).

[12] W.D. Mac Clement, M. Pintar, H.E. Petch, Can. J. Phys. 45, 3257 (1967).

[13] R.R. Knispel, H.E. Petch, Can. J. Phys. 49, 870 (1971).

[14] B. Hilczer, M. Pukacka, A. Sabat, Fiz. Dielektr. Radiospektr. XI, 69 (1979).

[15] K.A. Thuomas, A. Eriksson, A. Lund, J. Magn. Reson. 37, 223 (1980). 
[16] G.S. Chandler, A.D. McLean, J. Chem. Phys. 71, 2175 (1979).

[17] R. Fantechi, G.A. Helcke, J. Chem. Soc. Faraday Trans. II 68, 924 (1972).

[18] V. Malatesta, D. Lindsay, E.C. Horswill, K.V. Ingold, Can. J. Chem. 52, 864 (1974).

[19] J.Q. Adams, J.R. Thomas, J. Chem. Phys. 39, 1904 (1963).

[20] S. Gale, B. Johnson, K. Hendrich, R. Knispel, J.W. Harrell, J. Chem. Phys. 75, 4742 (1981).

[21] F. Köksal, O. Çakir, I. Gümrükçü, M. Birey, Z. Nat.forsch. A 40, 903 (1985).

[22] E. Sagstuen, O. Awadelkarim, A. Lund, J. Masiakowski, J. Chem. Phys. 85, 3223 (1986).

[23] B.H.J. Bielski, J.M. Gębicki, Atlas of ESR Spectra, Academic Press, New York 1967.

[24] A. Aharoni, J. Appl. Phys. 57, 4702 (1985).

[25] R. Durny, S. Yamasaki, J. Isoya, A. Matsuda, K. Tanaka, J. Non-Cryst. Solids 164-166, 223 (1993).

[26] M. Gramza, W. Hilczer, J. Goslar, S.K. Hoffmann, Acta Chem. Scand., 1997, in press.

[27] L.R. Dalton, A.L. Kwiran, J.A. Cowen, Chem. Phys. Lett. 17, 495 (1972).

[28] S.K. Iloffmann, W. Hilczer, J. Goslar, J. Magn. Reson. A 122, 37 (1996).

[29] M.K. Bowman, L. Kevan, in: Time Domain Electron Spin Resonance, Eds. L. Kevan, R.N. Schwartz, Wiley, New York 1979, Ch. 3.

[30] J. Murphy, Phys. Rev. 145, 241 (1966).

[31] V.A. Ivanshin, I.N. Kurkin, W. Brunner, G. Voelkel, Phys. Status Solidi B 151, K53 (1989). 